\title{
Automatic Non-rigid Temporal Alignment of IVUS Sequences
}

\author{
Marina Alberti ${ }^{1,2}$, Simone Balocco ${ }^{1,2}$, Xavier Carrillo ${ }^{3}$, \\ Josepa Mauri ${ }^{3}$, and Petia Radeva ${ }^{1,2, \star}$ \\ 1 Dept. of Applied Mathematics and Analysis, University of Barcelona, Spain \\ 2 Computer Vision Center, Campus UAB, Bellaterra, Barcelona, Spain \\ 3 University Hospital "Germans Trias i Pujol", Badalona, Spain \\ malberti@cvc.uab.es
}

\begin{abstract}
Clinical studies on atherosclerosis regression/progression performed by Intravascular Ultrasound analysis require the alignment of pullbacks of the same patient before and after clinical interventions. In this paper, a methodology for the automatic alignment of IVUS sequences based on the Dynamic Time Warping technique is proposed. The method is adapted to the specific IVUS alignment task by applying the non-rigid alignment technique to multidimensional morphological signals, and by introducing a sliding window approach together with a regularization term. To show the effectiveness of our method, an extensive validation is performed both on synthetic data and in-vivo IVUS sequences. The proposed method is robust to stent deployment and post dilation surgery and reaches an alignment error of approximately $0.7 \mathrm{~mm}$ for in-vivo data, which is comparable to the inter-observer variability.
\end{abstract}

\section{Introduction}

Intravascular Ultrasound (IVUS) is a catheter-based imaging technique used for diagnostic purposes and for planning and validation of Percutaneous Coronary Intervention (PCI). IVUS sequences are acquired by dragging an ultrasound emitter carried by a catheter, at constant speed, inside the arterial vessel, from the distal to the proximal position (pullbacks). The image alignment of the same vessel from different IVUS pullbacks is important from a clinical viewpoint. After performing PCI, frame alignment allows physicians to assess the intervention outcome (i.e., evaluate final lumen dimensions and blood flow restoration, detect stent malapposition and inspect side-branch occlusion by deployed stent). At follow-up, it is crucial to monitor restenosis and the evolution of plaque composition. Currently, in plaque regression studies, the longitudinal correspondence of coronary artery segments is manually determined by identifying common landmarks, such as bifurcations [1, 2]. To our knowledge, no method for the automatic alignment of IVUS sequences has been published in literature.

\footnotetext{
* This work is supported by TIN2009-14404-C02 and CONSOLIDER INGENIO CSD 2007-00018.
} 
Pullback 1

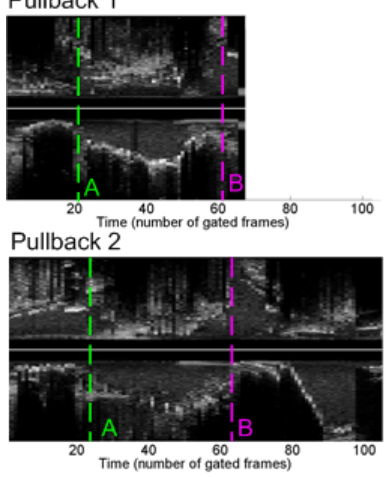

(a)
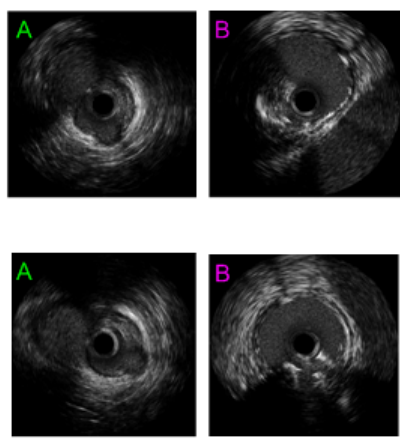

(b)
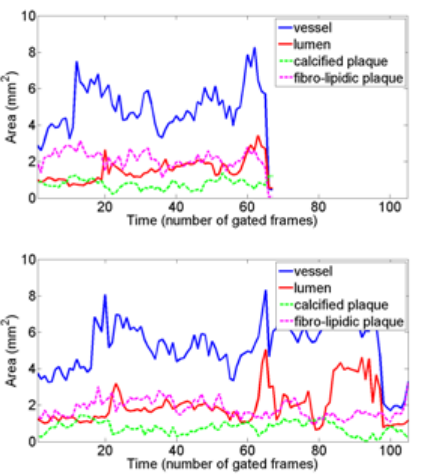

(c)

Fig. 1. Pair of IVUS sequences of the same vessel: (a) longitudinal views, (b) corresponding frames, (c) temporal signals describing the pullbacks

Despite the constant catheter speed, an automatic alignment is hampered by several obstacles: (1) the heart beating causes a non-rigid deformation of the vessel, (2) the catheter and the heart motion generate acquisition artifacts, such as the catheter longitudinal swinging and the roto-translation of successive frames (see Figure 1(b)), (3) the catheter can follow different trajectories inside the vessel, therefore the acquired image sections might not be orthogonal to the vessel walls, (4) the probe can remain stuck in the vessel for some time and then accelerate, (5) different initial and end spatial positions of the pullbacks cause partial overlapping (see Figure 1(a)) and (6) the vessel morphology can significantly change after the intervention or evolve at follow-up. Consequently, there is no one-to-one correspondence between frames of the two pullbacks, thus making image-based alignment approaches inaccurate. Hence, instead of using an image-based description, in this study the morphological content of the artery is exploited. IVUS sequences can be described by temporal morphological signals, i.e., side-branch location, vessel, lumen and plaque areas (see Figure1(c)). Thus, the IVUS alignment task is addressed as a temporal alignment problem.

In different applications, such as speech recognition, activity recognition, shape matching, several methods have been developed for non-rigid signal alignment, like Dynamic Time Warping (DTW), Canonical Time Warping (CTW) and Correlation Optimized Warping (COW) [3 5]. DTW [3] minimizes the Euclidean distance of corresponding points of the signals. Canonical Time Warping (CTW) [4] improves DTW by combining it with Canonical Correlation Analysis (CCA), thus adding a feature weighting mechanism. The optimization process requires the application of $\mathrm{CCA}$ at successive iterations. Correlation Optimized Warping (COW) [5] is a piecewise data alignment method which allows limited changes in segment lengths and performs a segment-wise correlation optimization by means of dynamic programming. 
To address the non-rigid correspondence between frames, in this paper a DTW-based framework is adapted to the specific clinical task and compared vs. the CTW and COW techniques. To tackle the partial overlapping problem, the alignment algorithms are integrated into a sliding window approach. Moreover, a regularization term is introduced to penalize significant differences in the global temporal expansion/compression of IVUS sequences. Two validations are presented, on synthetic data and on 13 pairs of in-vivo sequences (26 pullbacks).

\section{Method for IVUS Sequences Alignment}

The signal alignment framework is based on the DTW technique. To align two sequences $X=\left[x_{1}, x_{2}, \ldots x_{n_{x}}\right]$ and $Y=\left[y_{1}, y_{2}, \ldots y_{n_{y}}\right]$, DTW builds a matrix $d_{\left(n_{x} \times n_{y}\right)}$, where $d(i, j)\left(1 \leq i \leq n_{x} ; 1 \leq j \leq n_{y}\right)$ represents a dissimilarity measure between $X(i)$ and $Y(j)$ [3]. In the classical DTW formulation, $d(i, j)$ is computed as the Euclidean distance $d_{E}(i, j)$. Successively, the algorithm finds the warping path (i.e., a mapping of the time axes of $X$ and $Y$ on a common time axis, $\mathbf{f}=\langle[i(k), j(k)] \mid k=1, \ldots, K\rangle)$ with the minimum cumulative distance (MCD). The MCD of the path leading to the entry $(i, j)$ is computed by dynamic programming as: $D(i, j)=d(i, j)+\min (D(i-1, j), D(i-1, j-1), D(i, j-1))$. Finally, the matching cost is calculated as $\Phi(X, Y)=\underset{\mathbf{f}}{\operatorname{argmin}} \sum_{k=1}^{K} d(i(k), j(k))$.

\subsection{Multidimensional Profiles Extraction}

Profile Framework: A pair of corresponding IVUS sequences is described by temporal morphological profiles (i.e., signals describing the evolution of morphological measurements along the vessel) and defined as a pair of time series $X \in \mathbb{R}^{m \times n_{x}}$ and $Y \in \mathbb{R}^{m \times n_{y}}$, of length $n_{x}, n_{y}$ and dimensionality $m$. The profiles are invariant to frame rotation, thus making the method independent from the catheter torsion. The use of multiple features is aimed to increase the robustness with respect to 1-D alignment. The following morphological measurements are proposed in this study: (1) vessel area, defined as the area inside the mediaadventitia border [6], (2) lumen area [7], (3) area of calcified plaque [8], (4) area of fibro-lipidic plaque [8], (5) angular extension of bifurcations [9]. It is worth noticing that the framework could potentially provide similar results using a different set of profiles and it is independent from the technique employed for the measurements.

Gating Preprocessing: To limit the morphological variations due to vessel pulsation and catheter swinging effect, an image-based gating algorithm [10] is applied to the pullbacks in order to select the frames belonging to the enddiastolic phase. The selected images provide coherent morphological measures, since the arterial tissues are subject to the same blood pressure. Moreover, the preprocessing assures that the frames are consecutive in the direction of the catheter movement. 


\subsection{IVUS Alignment Framework}

Sliding Window (SW) Approach: The DTW approach suffers from a limitation: it attempts to compute a global matching between all the frames of the two sequences, even in presence of partial overlapping (see Figure 1(a)). Hence, the alignment algorithm is integrated into a sliding window approach. The two sequences are iteratively slided one along the other and for each step the alignment between the overlapping subsegments is identified. The optimal iteration is selected by minimizing a matching cost $\Phi_{N O R M}\left(X_{i t e r}, Y_{\text {iter }}\right)=$ $\Phi\left(X_{i t e r}, Y_{i t e r}\right) / l_{i t e r}$, where $X_{i t e r}$ and $Y_{i t e r}$ are the overlapping subsegments and $l_{\text {iter }}$ is the length of the overlapping window at iteration iter. In order to decrease the computational cost, the allowed overlap ranges from $n_{s}-w_{\text {elast }}$ to $n_{s}$, where $n_{s}$ is the length of the shortest sequence.

Regularization Cost (RC): In the classical version of DTW [3], an arbitrary band constraint is employed to guide the warping path by limiting its acceptable domain to a band around the diagonal of the dissimilarity matrix $d$. In this paper, a regularization term, described by a continuous formulation, is introduced to favor warping paths closer to the diagonal. Such improvement automatically penalizes an excessive and non-physiological compression/expansion of the two sequences. In the $D T W+S W$ framework, the dissimilarity measure $d(i, j)$ is computed as: $d(i, j)=d_{E}(i, j)+w_{R} \cdot d_{R}(i, j)$, where $d_{R}(i, j)=|i-j|$ is the regularization cost (see Figure 2(d)) and $w_{R}$ represents the penalization weight. The regularization is aimed to reduce the alignment error when the profiles are affected by noise corruption, as shown in Figure 2(a)-(c).

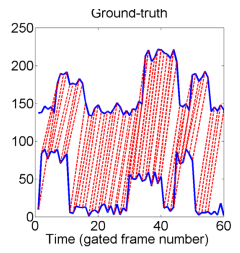

(a)

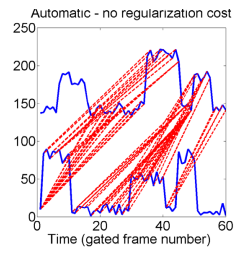

(b)

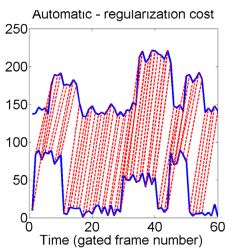

(c)

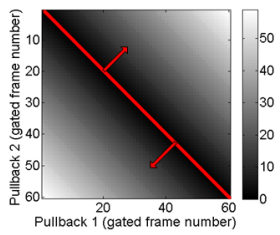

(d)

Fig. 2. Example of alignment of noise-corrupted signals. In (b) incorrect alignment by classical DTW, in (c) correct alignment by applying $\mathrm{RC}$, in (d) regularization cost matrix $d_{R}$, where the red arrows indicate the penalization increase. In (a), (b), (c), blue lines represent the signals and red lines represent correspondences between frames.

\section{$3 \quad$ Experimental Results}

Our dataset consists of 26 in-vivo IVUS sequences from human coronary arteries. The acquisitions have been performed both preoperatively and after intervention (stent deployment, post dilation), by using an iLab IVUS Imaging System (Boston Scientific). The performance of DTW is fairly compared with two other 
state-of-the-art techniques, CTW and COW, (1) adapting the classical formulation to multidimensional signals, using the same weight for the different features, (2) integrating into the SW approach and (3) additionally applying the regularization term $(S W+R C)$. It is worth noticing that both DTW and CTW are fully automatic, while COW requires the setting of two parameters, the initial segment length $S e g$ and the maximum segment length variation Slack. Additionally, two new parameters related to the developed IVUS framework are introduced for each alignment method, $w_{\text {elast }}$ and $w_{R}$, for SW and RC, respectively. The alignment error $E$ is defined as the average distance between the reference and the output warping path, expressed in number of gated frames.

\subsection{Experiments on Synthetic Data}

Synthetic Morphological Signals: Pairs of sequences $(X, Y)$ are synthetically generated by modifying the morphological profiles extracted from the 26 in-vivo pullbacks. The scheme in Figure 3 summarizes the applied types of distortion:

1. Amplitude distortion: additive zero-mean random noise is applied to the morphological profiles. The noise amplitude $w_{1}$ is computed as a percentage of the mean value of the signal (Figure 3 (a)).

2. Partial overlapping: a portion of the original sequence is selected, whose length is a percentage $w_{2}$ of the initial profile (Figure 3(b)).

3. Temporal distortion: a temporal expansion/compression generates vertical or horizontal transitions in the warping path, i.e., multiple correspondences between the frames of $X$ and $Y$. We distinguish three cases, in which we randomly introduce: (a) the same number $\left(w_{3}\right)$ of multiple correspondences from $X$ to $Y$ and vice-versa (Figure 3(c)); (b) additional multiple correspondences from $X$ to $Y$ (Figure 3 (d)); (c) additional multiple correspondences from $Y$ to $X$ (Figure $3(\mathrm{e})$ ).

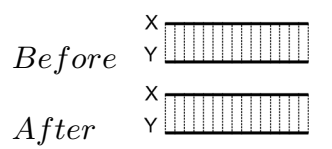

(a)

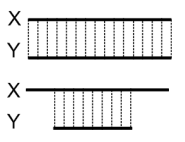

(b)

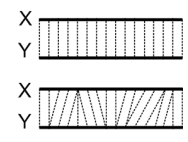

(c)

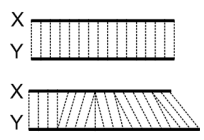

(d)

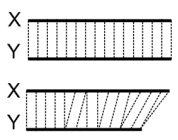

(e)

Fig. 3. Idealized pairs of sequences $(X, Y)$ (continuous lines), and frame-to-frame correspondences (dotted lines), before and after the distortion simulation

The parameters $w_{1}, w_{2}, w_{3}, w_{4}, w_{5}$ model the signal distortion simulation. Their default values and ranges, which represent average in-vivo conditions and realistic variations, respectively, are suggested by a medical expert and empirically measured on the whole ground-truth: $w_{1}=(100 \pm 100) \%, w_{2}=(75 \pm 25) \%$, $w_{3}=60(0-120)$ frames, $w_{4}=5(0-15)$ frames, $w_{5}=0(0-15)$ frames. The tuning of the parameters of the alignment methods, $w_{\text {elast }}, w_{R}$, Seg, Slack, is performed by minimizing the mean of $E$ over $N_{\text {iter }}=40$ experiments, setting 
Table 1. Quantitative results $(M E A N \pm S T D)$ for $E$ on synthetic data, as a function of $m$, and on in-vivo data. For the in-vivo experiments, bifurcation profiles are not used.

\begin{tabular}{|l||c|c|c|c|c||c|}
\hline \multicolumn{1}{|c||}{} & \multicolumn{5}{c|}{ Synthetic } & In - vivo \\
\hline & $m=1$ & $m=2$ & $m=3$ & $m=4$ & $m=5$ & $m=4$ \\
\hline \hline$D T W$ & $9.21 \pm 9.28$ & $4.37 \pm 3.89$ & $2.78 \pm 2.85$ & $1.88 \pm 0.93$ & $1.5 \pm 0.58$ & $3 \pm 2.68$ \\
\hline$D T W+S W$ & $7.05 \pm 9.31$ & $3.38 \pm 4.84$ & $2.04 \pm 3.49$ & $1.21 \pm 0.68$ & $0.96 \pm 0.5$ & $3.29 \pm 5.16$ \\
\hline$D T W+S W+R C$ & $3.98 \pm 5.09$ & $1.98 \pm 1.65$ & $1.37 \pm 0.71$ & $1.1 \pm 0.51$ & $\mathbf{0 . 9 3} \pm \mathbf{0 . 4 4}$ & $\mathbf{1 . 4 3} \pm \mathbf{0 . 6 8}$ \\
\hline \hline$C T W$ & $9.57 \pm 9.39$ & $4.33 \pm 3.72$ & $2.79 \pm 2.3$ & $2.14 \pm 0.93$ & $1.8 \pm 0.74$ & $3.94 \pm 3.91$ \\
\hline$C T W+S W$ & $6.59 \pm 8.95$ & $2.62 \pm 2.48$ & $1.76 \pm 0.91$ & $1.38 \pm 0.66$ & $1.06 \pm 0.47$ & $2.54 \pm 1.96$ \\
\hline$C T W+S W+R C$ & $5.72 \pm 7.75$ & $2.29 \pm 2.2$ & $1.64 \pm 0.99$ & $1.29 \pm 0.57$ & $\mathbf{1 . 0 7} \pm \mathbf{0 . 4 6}$ & $\mathbf{1 . 6 2} \pm \mathbf{0 . 8 2}$ \\
\hline \hline$C O W$ & $8.77 \pm 3.91$ & $9.69 \pm 3.86$ & $9.71 \pm 4.02$ & $9.85 \pm 4.29$ & $9.7 \pm 4.34$ & $7.54 \pm 5.61$ \\
\hline$C O W+S W$ & $4.4 \pm 5.31$ & $2.6 \pm 1.38$ & $2.35 \pm 1.07$ & $2.36 \pm 1.01$ & $2.41 \pm 0.93$ & $2.68 \pm 1.71$ \\
\hline$C O W+S W+R C$ & $4.4 \pm 5.17$ & $2.53 \pm 1.48$ & $2.24 \pm 0.89$ & $2.21 \pm 0.92$ & $\mathbf{2 . 3} \pm \mathbf{1 . 2 2}$ & $\mathbf{2 . 4 6} \pm \mathbf{1 . 5}$ \\
\hline \hline inter - observer & \multicolumn{7}{|c||}{} & & $\mathbf{1 . 2} \pm \mathbf{1 . 4 1}$ \\
\hline
\end{tabular}

the distortion parameters to default values. The parameters are estimated by exhaustive search: $w_{\text {elast }} \in[0,35], w_{R} \in[0,0.003]$ for DTW $/$ CTW, $w_{R} \in[0,0.1]$ for COW, Seg $\in[16,30]$ and Slack $\in[6$, Seg -9$]$.

Relevance of the Multidimensional Alignment: In order to evaluate the robustness of the framework as a function of the number of morphological profiles, $E$ is computed over $N_{\text {iter }}=40$ experiments, for $m=1, \ldots, 5$. Pairs of synthetic signals are generated by setting $w_{1}, w_{2}, w_{3}, w_{4}, w_{5}$ to default values. As expected, Table 1 shows that $E$ decreases as $m$ increases. The error reduction is particularly high when more than one signal are considered, confirming the interest of a multidimensional extension of the method.

Feature Relevance Estimation: From the previous experiment, the relevance of the different morphological profiles is assessed. To this aim, the feature occurrence in the optimal feature combinations, minimizing $E$, is determined. The following rank for relevance is obtained: (1) calcified plaque area, (2) bifurcation extension, (3) fibro-lipidic plaque area, (4) lumen area, (5) vessel area.

Robustness to Signal Noise and Distortion: In the second set of experiments, the robustness to noise and distortion is evaluated (see Figure 4). When one of the distortion parameters is varied in the chosen range, the others are set to default values. This experiment demonstrates that the SW approach is useful since DTW, CTW and COW are robust to partial overlapping only when integrated in the SW framework (see Figure 4 (first row, middle)). SW and RC are generally advantageous (see Figure 4 (first row)) except when $w_{4}$ and $w_{5}$ are very high (see Figure 4 (second row)). COW always shows the highest error, indicating that a segment-wise alignment is not suited for the IVUS problem. 

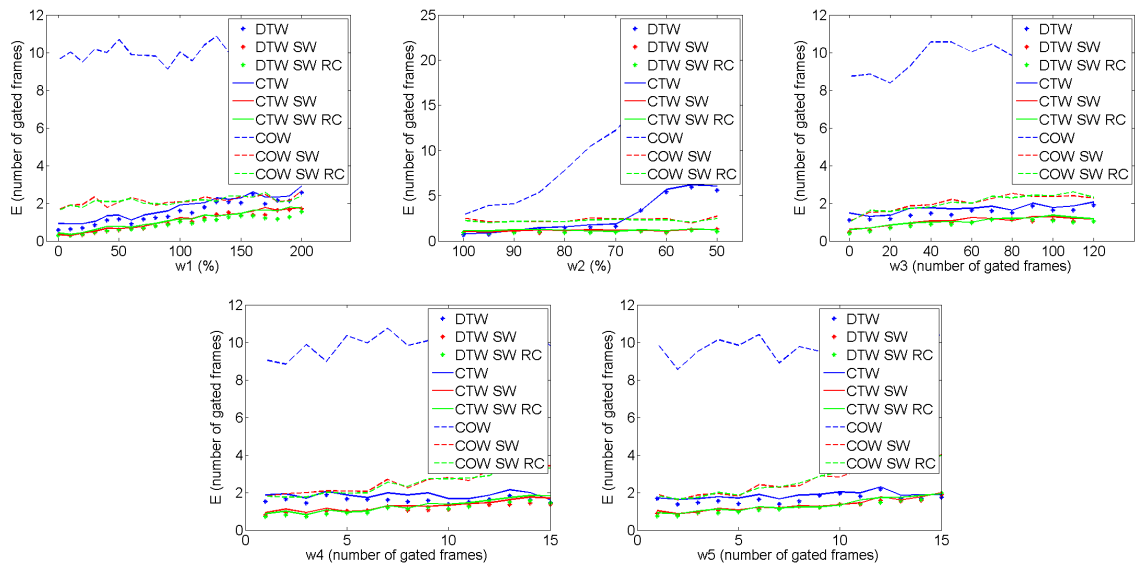

Fig. 4. $E$ as a function of amplitude distortion $\left(w_{1}\right)$, partial overlapping $\left(w_{2}\right)$, temporal distortions $\left(w_{3}, w_{4}, w_{5}\right)$
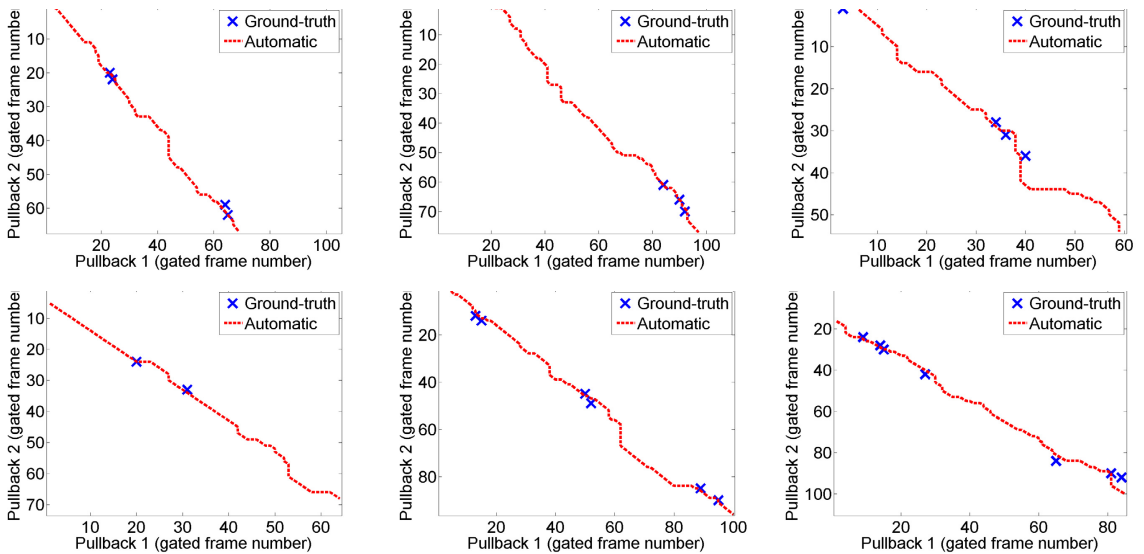

Fig. 5. Ground-truth and automatic warping path, computed by $D T W+S W+R C$

\subsection{Experiments on In-vivo Data}

The performance of the proposed approach is evaluated over 13 pairs of corresponding pullbacks, all characterized by morphological changes due to intervention. Following the same strategy employed in [1], corresponding bifurcation locations (initial and end positions) are used as ground-truth. Manual annotations are performed by two physicians. Indeed side branches are the only immutable landmarks, since lumen and media size, stent and plaque might vary due to surgical artery dilatation or stent deployment. Hence, in this experiment, the signal of bifurcation extension is excluded from the alignment framework. The parameter tuning is performed by means of Leave-One-Patient-Out (LOPO) tech- 
nique: given the number of correspondences, one of them is iteratively used as test set, and the parameters are optimized by minimizing $E$ over all the other correspondances. As observed in Table 1 (seventh column), the $S W+R C$ framework always reaches the lowest error. The Wilcoxon test proves that with a significance level $\alpha=0.1$, the performance of $D T W+S W+R C$ is significantly better than $C O W+S W+R C$ and comparable to $C T W+S W+R C$ and to the inter-observer variability. The mean value for $E$ by $D T W+S W+R C-1.43$ gated frames - can be estimated around $0.7 \mathrm{~mm}$. In Figure 5 , ground-truth and output warping paths for pairs of corresponding pullbacks are illustrated. The only significant errors are close to the extremes of the sliding window (Figure 5 (third column)).

\section{Discussion and Conclusion}

This paper presents, for the first time, a fully automatic framework for the temporal alignment of IVUS pullbacks. The proposed method is robust to morphological changes induced by stent deployment and post dilation, and is invariant to the catheter or imaging system employed. Extensive experiments show that the use of multiple morphological profiles along with the sliding window approach and regularization term enhance the result. Future work will address the application of this framework on intra-modality alignments, for instance, IVUS and Optical Coherence Tomography sequences. Furthermore, the proposed method will be evaluated on follow up cases. Finally, the application of feature weights based on the feature relevance will be investigated.

\section{References}

1. Diletti, R., Garcia-Garcia, H.M., Gomez-Lara, J., Brugaletta, S., Wykrzykowska, J.J., van Ditzhuijzen, N., van Geuns, R.J., Regar, E., Ambrosio, G., Serruys, P.W.: Assessment of coronary atherosclerosis progression and regression at bifurcations using combined ivus and oct. JACC Cardiovasc. Imaging 4, 774-780 (2011)

2. Kovarnik, T., Wahle, A., Downe, R.W., Sonka, M.: IVUS Role in Studies Assessing Atherosclerosis Development. In: Intrvascular Ultrasound, pp. 53-68. InTech (2012)

3. Sakoe, H., Chiba, S.: Dynamic programming algorithm optimization for spoken word recognition. IEEE TASSP 26, 43-49 (1978)

4. Zhou, F., de la Torre, F.: Canonical time warping for alignment of human behavior. In: NIPS, pp. 2286-2294 (2009)

5. Nielsen, N.P.V., Carstensen, J.M., Smedsgaard, J.: Aligning of single and multiple wavelength chromatographic profiles for chemometric data analysis using correlation optimised warping. Journal of Chromatography 805, 17-35 (1998)

6. Ciompi, F., Pujol, O., Gatta, C., Carrillo, X., Mauri, J., Radeva, P.: A Holistic Approach for the Detection of Media-Adventitia Border in IVUS. In: Fichtinger, G., Martel, A., Peters, T. (eds.) MICCAI 2011, Part III. LNCS, vol. 6893, pp. 411-419. Springer, Heidelberg (2011)

7. Balocco, S., Gatta, C., Ciompi, F., Pujol, O., Carrillo, X., Mauri, J., Radeva, P.: Combining Growcut and Temporal Correlation for IVUS Lumen Segmentation. In: Vitrià, J., Sanches, J.M., Hernández, M. (eds.) IbPRIA 2011. LNCS, vol. 6669, pp. 556-563. Springer, Heidelberg (2011) 
8. Ciompi, F., Pujol, O., Gatta, C., Leor, O.R., Ferre, J.M., Radeva, P.: Fusing in-vitro and in.vivo intravascular ultrasound data for plaque characterization. International Journal of Cardiovascular Imaging 26, 763-779 (2010)

9. Alberti, M., Gatta, C., Balocco, S., Ciompi, F., Pujol, O., Silva, J., Carrillo, X., Radeva, P.: Automatic Branching Detection in IVUS Sequences. In: Vitrià, J., Sanches, J.M., Hernández, M. (eds.) IbPRIA 2011. LNCS, vol. 6669, pp. 126-133. Springer, Heidelberg (2011)

10. Gatta, C., Balocco, S., Ciompi, F., Hemetsberger, R., Leor, O.R., Radeva, P.: RealTime Gating of IVUS Sequences Based on Motion Blur Analysis: Method and Quantitative Validation. In: Jiang, T., Navab, N., Pluim, J.P.W., Viergever, M.A. (eds.) MICCAI 2010, Part II. LNCS, vol. 6362, pp. 59-67. Springer, Heidelberg (2010) 